\title{
Nanoparticle-induced rectification in a single cylindrical nanopore: Net currents from zero time-average potentials
}

\author{
Mubarak Ali, ${ }^{1,2, a)}$ Patricio Ramirez, ${ }^{3}$ Saima Nasir, ${ }^{1,2}$ Quoc-Hung Nguyen, ${ }^{1,2}$ \\ Wolfgang Ensinger, ${ }^{1}$ and Salvador Mafe ${ }^{4, a)}$ \\ ${ }^{1}$ Department of Material-and Geo-Sciences, Technische Universität Darmstadt, D-64287 Darmstadt, \\ Germany \\ ${ }^{2}$ Materials Research Department, GSI Helmholtzzentrum für Schwerionenforschung, D-64291 Darmstadt, \\ Germany \\ ${ }^{3}$ Departament de Física Aplicada, Universitat Politécnica de València, E-46022 València, Spain \\ ${ }^{4}$ Departament de Física de la Terra i Termodinàmica, Universitat de València, E-46100 Burjassot, Spain
}

(Received 13 December 2013; accepted 15 January 2014; published online 29 January 2014)

\begin{abstract}
Rectification in nanopores is usually achieved by a fixed asymmetry in the pore geometry and charge distribution. We show here that nanoparticle blocking of a cylindrical pore induces rectifying properties that can support significant net currents with zero time-average potentials. To describe experimentally this effect, the steady-state current-voltage curves of a single nanopore are obtained for different charge states and relative sizes of the pore and the charged nanoparticles, which are present only on one side. The rectification phenomena observed can find applications in the area of nanofluidics and involves physical concepts that are also characteristic of the blocking of protein ion channels by ionic drugs. (C) 2014 AIP Publishing LLC. [http://dx.doi.org/10.1063/1.4863511]
\end{abstract}

Net currents can be obtained with zero time-average forces in spatially asymmetric systems, ${ }^{1-3}$ e.g., the ion channels inserted in biological membranes and nanofluidic diodes. ${ }^{4-7}$ We have recently shown this effect in the pumping of potassium ions against an external concentration gradient imposed on the bacterial porin $O m p F$ (outer membrane protein F) channel of Escherichia coli ${ }^{5}$ as well as in a single asymmetric nanopore functionalized with amino acid groups. ${ }^{4}$ We demonstrate in this Letter nanoparticle-induced rectification in a cylindrical pore using fundamental concepts of nanofluidics.

Nanopores track-etched in polymer films are more robust than ion channels and can be functionalized with electrically active moieties whose charge can be modulated externally through electrical, thermal, chemical, and optical signals, resulting in liquid-state transistors useful for separation, energy conversion, and chemical information processing. ${ }^{8-12}$ We are concerned with a nanoparticle-induced gating that supports rectification in a cylindrical nanopore (Fig. 1), as opposed to the usual case of an asymmetric pore geometry and charge distribution. ${ }^{4,71-14}$ The nanopore is symmetric but the system as a whole is not completely symmetric because the charged nanoparticles are present only on one side of the pore.

Nanoparticle blocking and release has previously been used for stochastic electrophoretic capture at the tip of conical pores using a scanning electrochemical microscope. ${ }^{14,15}$ However, our aim here is not to obtain the particle characteristics from the individual blocking and release events recorded in the current traces of an asymmetric membrane. ${ }^{14,15}$ Rather, we will show that net currents can result from zero time-average forces in a single cylindrical nanopore because of partial blocking of the pore. We demonstrate

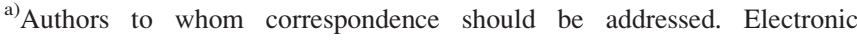
addresses: M.Ali@gsi.de and smafe@uv.es.
}

this effect for different charges and relative sizes of the nanostructures (see Fig. 1) allowing significant blocking for small pores $(r>R)$ and particle translocation for large pores $(r<R)$. The obvious case $r \gg R$ was not considered because we wanted to show that it is possible to tune the pore diameters in the experimental range $R_{\min }<r<R_{\max }$ and obtain significant blocking and rectification effects.

The results are of interest not only to nanofluidics but also to biophysics because protein ion channels can be blocked by ionic drugs. ${ }^{16-20}$ Although the local interactions in the channel are highly specific, the significant role of the applied voltage and the electrolyte concentration (Debye screening) strongly suggests that long range electrostatic interactions are also important. This fact has been observed in the binding of antibiotic molecules to bacterial ion channels,${ }^{16,17}$ the blocking of channels involved in toxin infections by highly charged drugs, ${ }^{18,19}$ and the conductance modulation of bacterial porin channels by trivalent ions. ${ }^{20}$ Electric potentials, rectification, and ratchet phenomena ${ }^{3}$ are also relevant in the signal averaging of low frequency electric fields by cell membranes because ion channels play a crucial role in cell growth and communication. ${ }^{21-24}$

Figures 1(a)-1(c) show the different charge states of the nanostructures and the low (high) current $I$ obtained for the blocked (unblocked) pore. Pore rectification depends on the sign of the applied voltage $V$. In Fig. 1(a), partial pore blocking $(V>0)$ and unblocking $(V<0)$ occur when the migration (MIG) flow resulting from the particle surface charge and the electro-osmotic (EOF) flow resulting from the pore surface charge act in concert, reinforcing the rectification effect. ${ }^{14,15,25}$ This mechanism is also shown in Fig. 1(b), with the trivial change in the sign of the blocking $(V<0)$ and unblocking $(V>0)$ voltages with respect to Fig. 1(a). On the contrary, migration opposes electro-osmosis when the nanoparticle and the pore surface have the same charge sign (Fig. 1(c)) regardless of the applied voltage. ${ }^{25}$ This fact, 
(a)

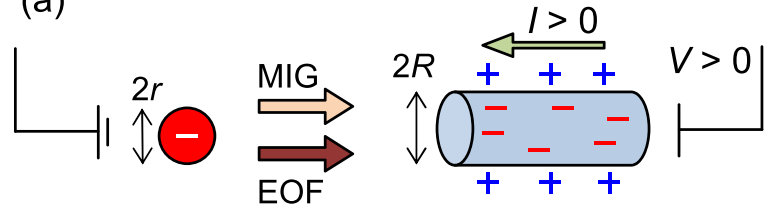

(b)

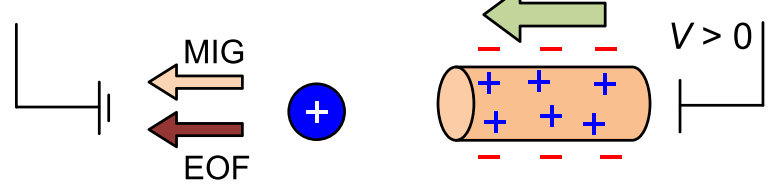

(c)

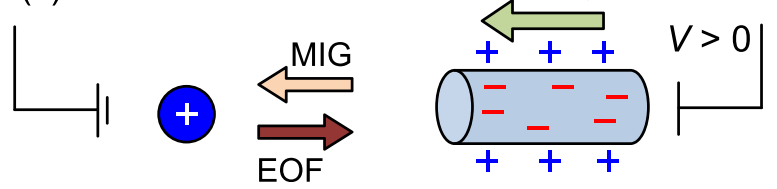

FIG. 1. Schemes of the charged nanoparticle and the cylindrical nanopore (a)-(c). The symbols inside the pore are the mobile charges compensating for the opposite charges fixed to the pore walls. Although the schemes correspond to the case $r \approx R$ only, we have considered the three experimental cases $r>R, r \approx R$, and $r<R$ for the nanoparticle and the pore radii in order to show the different pore blocking and rectification effects. The arrows show the current $I>0$ and its relative value for voltage $V>0$ (the ground electrode is on the left side of the pore where the charged nanoparticles are added). The electro-osmotic and migration flows at the pore entrance are drawn out of the pore for the sake of clarity and should be reversed when the voltage is reversed $(V<0)$. Partial pore blocking occurs for $V>0$ in Fig. 1(a) and $V<0$ in Fig. 1(b) while this effect is inhibited in Fig. 1(c) because the electro-osmotic and migration terms tend to cancel one another and the nanoparticle is electrostatically excluded from the pore.

together with the repulsive electrostatic force between the nanoparticle and the pore, inhibits rectification in Fig. 1(c). Note that models for blocking predict high electric fields in the solution close to the pore mouth. ${ }^{14,19,25}$

Single, approximately cylindrical pores are obtained in $12 \mu \mathrm{m}$ thick polyethylene terephthalate membranes using track-etching procedures. ${ }^{8,10,26}$ Three samples symmetrically etched for 5, 10, and $15 \mathrm{~min}$ give the average pore radii $R=15,30$, and $50 \mathrm{~nm}$ approximately (Figs. 2(a)-2(c) and $3(\mathrm{a})-3(\mathrm{c}))$, as determined from the linear $I-V$ curves of Figs. 2(a) and 3(a). ${ }^{10}$ The pore is functionalized either with carboxylic $(\mathrm{COOH})$ or amine $\left(\mathrm{NH}_{2}\right)$ groups that are ionized $\left(\mathrm{COO}^{-}\right.$and $\left.\mathrm{NH}_{3}{ }^{+}\right)$at $p \mathrm{H}=5.8$. Polystyrene (PS) nanoparticles PS-COOH and PS- $\mathrm{NH}_{2}$ of radius $\sim 30 \mathrm{~nm}$, as provided by the supplier, are added on left side of the pore at a concentration of $10^{12}$ particles $\mathrm{ml}^{-1}$, approximately. The two bathing solutions contain the electrolyte $\mathrm{KCl}$ at the same molar concentration $c$ in $0.01 \% \mathrm{v} / \mathrm{v}$ Tween 20 surfactant. The quasi steady-state $I$ - $V$ curves measured do not depend on the potential scan rate at the low frequency used in Figs. 2-4 (see Ref. 10 for additional experimental details).

Figures 2(a)-2(c) show the $I-V$ curves for the negatively charged nanoparticle and three positively charged pores
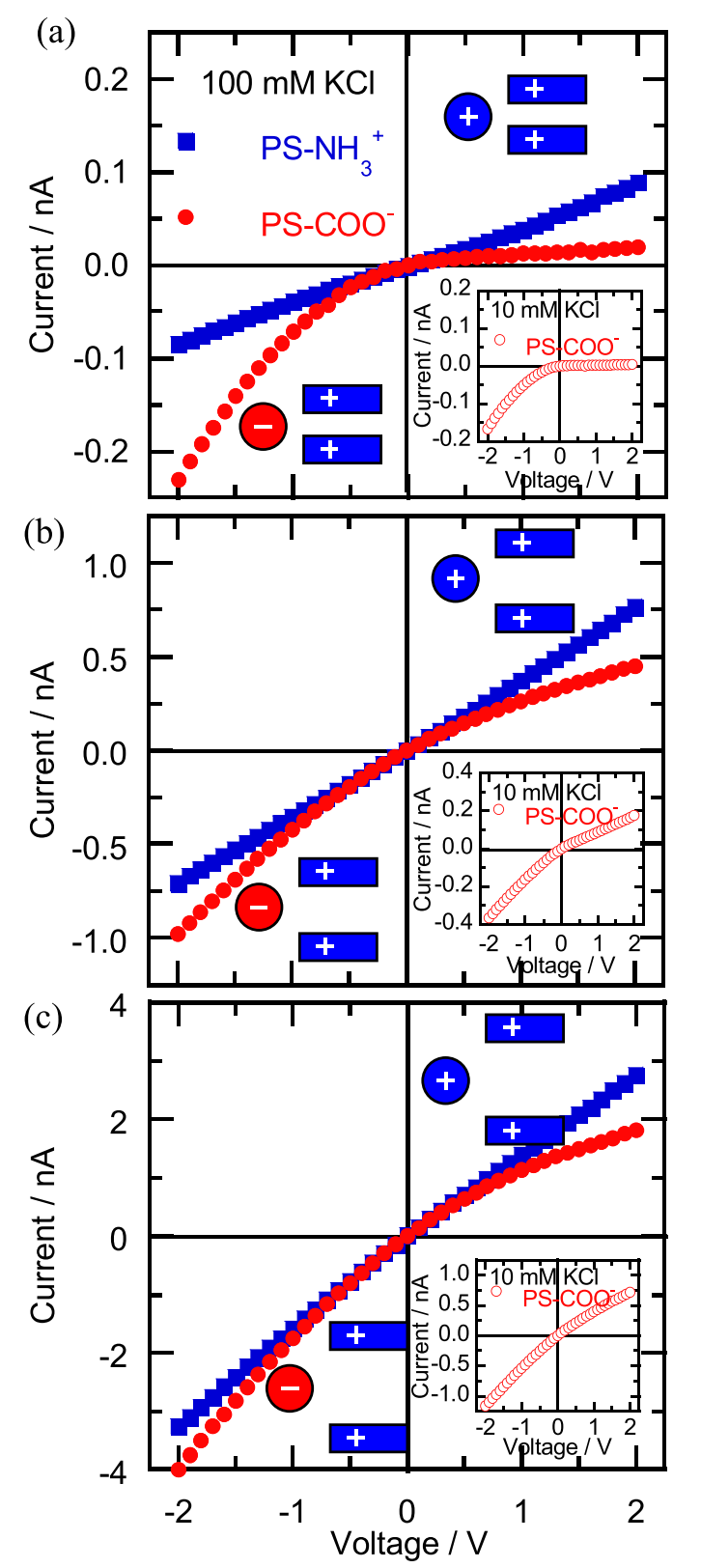

FIG. 2. I-V curves for the negatively charged PS-COO ${ }^{-}$nanoparticle $(r=30 \mathrm{~nm})$ and the positively charged pores (Fig. 1(a)) of approximate radii $R=15$ (a), 30(b), and $50 \mathrm{~nm}$ (c). The linear $I-V$ curve (no rectification) obtained for the positively charged PS- $\mathrm{NH}_{3}{ }^{+}$nanoparticle $(r=30 \mathrm{~nm})$ shows clearly that blocking is inhibited in Fig. 1(c), suggesting also that the positive pore is approximately symmetrical. The insets show the effect of decreasing the $\mathrm{KCl}$ concentration from $c=100 \mathrm{mM}$ to $10 \mathrm{mM}$ at $p \mathrm{H}=5.8$.

(Fig. 1(a)) of different radii. The case of the positively charged nanoparticle gives linear $I-V$ curves (no rectification) because of the absence of pore blocking (Fig. 1(c)). The rectification increases with decreasing the pore radius and the electrolyte concentration (insets) because the decreasing of the Debye screening enhances the electrostatic focusing ${ }^{27}$ of the nanoparticle to the pore.

Figures 3(a)-3(c) show the $I-V$ curves for the positively charged nanoparticle and the three negatively charged pores (Fig. 1(b)) of different radii at two $\mathrm{KCl}$ concentrations. Figure 3(a) gives a high current in presence of the nanoparticle, presumably because of the locally high ionic concentration around the charged nanoparticles at the pore mouth. ${ }^{28}$ 

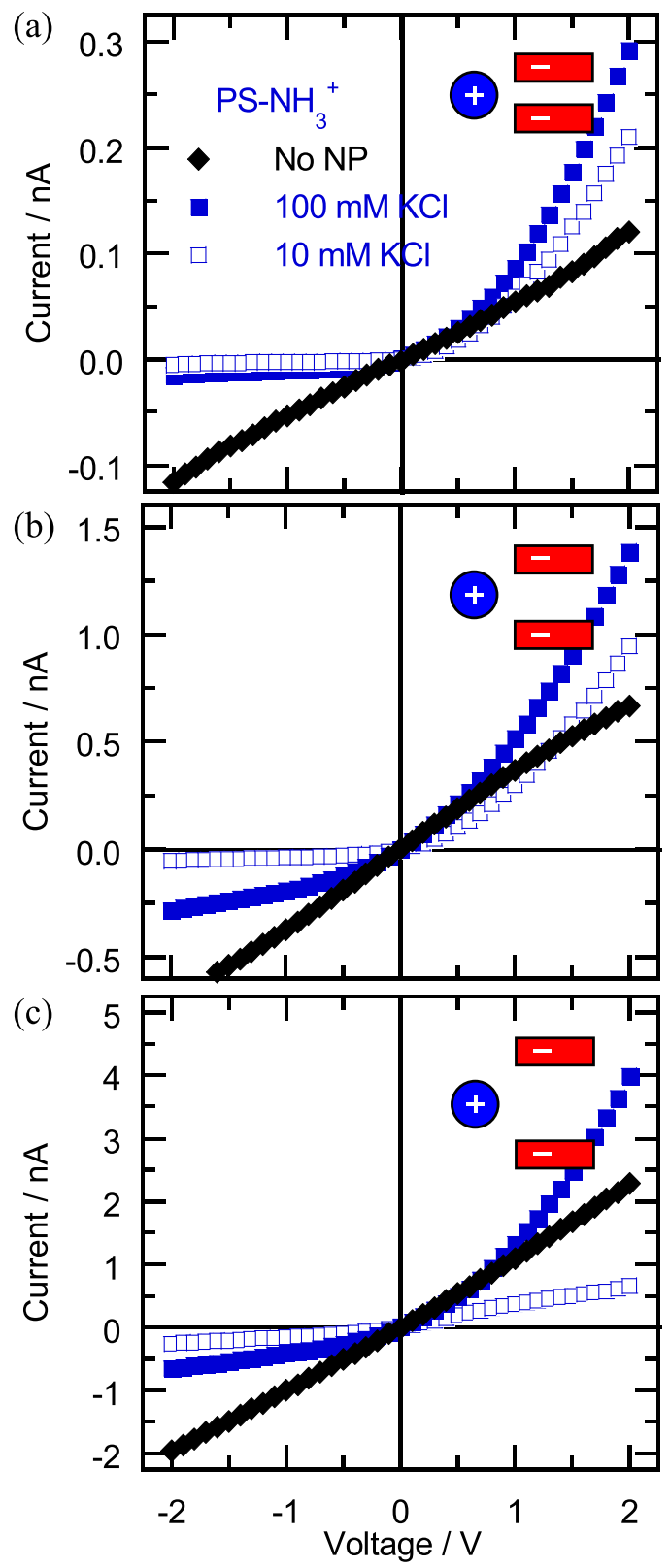

FIG. 3. $I-V$ curves for the positive nanoparticle and the negative pores (Fig. 1(b)) of different radii parametrically in $\mathrm{c}$ at $p \mathrm{H}=5.8$ (a)-(c). The linear $I-V$ curve obtained in absence of nanoparticle (No NP) shows that the negative pore is also symmetrical.

These particles have radii similar to that of the pore and behave then as effective outer pore walls whose high charge attracts the small salt ions. ${ }^{29}$ At low salt concentration, these mobile ions may compensate for the diffusion-limited conductance at the pore entrance. Note also that the quasi-linear $I-V$ curves obtained in absence of nanoparticle (Fig. 3(a)) and blocking (Fig. 2(a)) confirm the similar characteristics of the two pores. Rectification should then be ascribed to blocking and is enhanced here by the large aspect ratio of the polymeric pore compared with solid-state nanopores. ${ }^{30}$

Figure 4(a) shows representative $I(t)$ vs. $t$ curves for zero time-average potentials $V(t)$ of different amplitudes $V_{0}$. At the low scan rates of Fig. 4(a), the instantaneous current $I(t)$ is slave of the input signal $V(t)$. The time average current $\langle I\rangle$ can therefore be obtained from the experimental currents of Figs. 2 and 3 assuming the adiabatic limit ${ }^{2,4,5}$ as $\langle I\rangle=\left[I\left(V_{0}\right)+I\left(-V_{0}\right)\right] / 2$. (a)

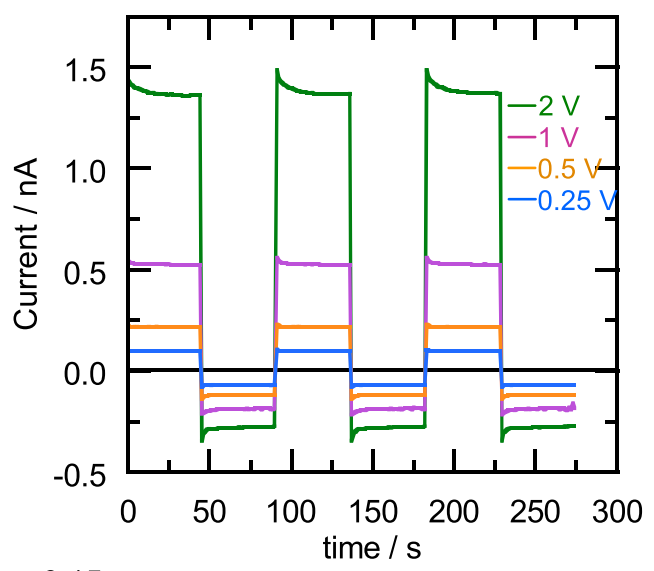

(b)

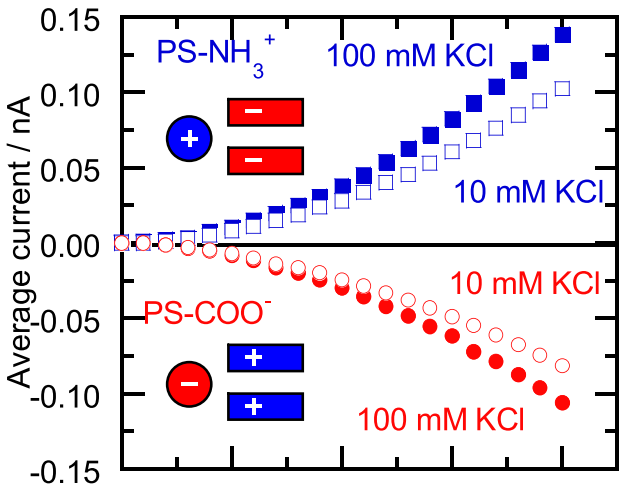

(c)

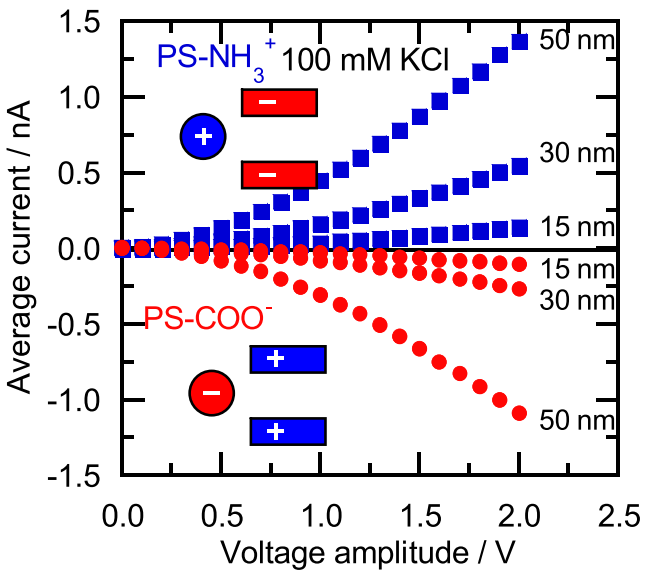

FIG. 4. Instantaneous current vs. time $t$ curves obtained for square wave voltages $V(t)$ of amplitudes $V_{0}=2,1,0.5$, and $0.25 \mathrm{~V}$ (a). The experimental data correspond to the positive particle and the negative pore of intermediate radius $R=30 \mathrm{~nm}$ at $c=100 \mathrm{mM}$ (Fig. 3(b)). The current $\langle I\rangle$ averaged over a signal period vs. $V_{0}$ parametrically in $c$ for $R=15 \mathrm{~nm}$ (b). The current $\langle I\rangle$ vs. $\mathrm{V}_{0}$ parametrically in $R$ for $c=100 \mathrm{mM}$ (c).

Figures 4(b) and 4(c) show the $\langle I\rangle$ vs. $V_{0}$ curves for different $\mathrm{KCl}$ concentrations and pore radii. The input driving potential averages zero over a time period but the average output current $\langle I\rangle$ is non-zero because of the rectification induced on the cylindrical nanopore, as shown previously in asymmetric ion channels ${ }^{5}$ and nanopores. ${ }^{4,31,32}$ As expected, the net currents increase with the pore radius $R$ and the electrolyte concentration $c$ (despite the fact that the rectification ratio decreases with $R$ and $c$ in Figs. 2 and 3) because of the increase in the pore area and the number of mobile ions available for transport. Note that the net average currents $\langle I\rangle$ are of similar order of magnitude as the currents $I$. These results could be enhanced in practical applications by using multi-pore membranes, ${ }^{33}$ allowing 
significant cumulative effects (e.g., charge storage) for long enough periods.

In conclusion, we have presented here an approach to achieve current rectification in cylindrical nanopores with symmetrical pore geometry and charge distribution (the asymmetry is imposed on the external solutions). The rectifying properties obtained can be used to produce significant net currents with zero time-average applied voltages. The fact that the nanoparticle-induced rectification is observed for different charge states and relative sizes of the nanostructures suggests that this effect is robust and can then be extended to other driving forces, switching the nanoparticlepore system between two states of different conductance. The rectification phenomena observed involve physical concepts that are also found in biological systems and can find applications in sensing, energy harvesting and nanofluidics.

M.A., S.N., Q.H.N., and W.E. acknowledge the BeilsteinInstitut, Frankfurt/Main, Germany, within the research collaboration NanoBiC. P.R. and S.M. acknowledge the Ministry of Economy and Competitiveness (project MAT2012-32084) and the Generalitat Valenciana (project PROMETEO/GV/0069). The authors thank Professor Christina Trautmann from GSI for support with the heavy ion irradiation experiments.

${ }^{1}$ R. D. Astumian and P. Hänggi, Phys. Today 55(11), 33 (2002).

${ }^{2}$ M. O. Magnasco, Phys. Rev. Lett. 71, 1477 (1993).

${ }^{3}$ P. Hänggi and F. Marchesoni, Rev. Mod. Phys. 81, 387 (2009).

${ }^{4}$ P. Ramirez, V. Gomez, M. Ali, W. Ensinger, and S. Mafe, Electrochem. Commun. 31, 137 (2013).

${ }^{5}$ M. Queralt-Martín, E. García-Giménez, V. M. Aguilella, P. Ramirez, S. Mafe, and A. Alcaraz, Appl. Phys. Lett. 103, 043707 (2013).

${ }^{6}$ Z. Siwy and A. Fuliński, Phys. Rev. Lett. 89, 198103 (2002).

${ }^{7}$ I. Vlassiouk and Z. Siwy, Nano Lett. 7, 552 (2007).

${ }^{8}$ Z. S. Siwy, Adv. Funct. Mater. 16, 735 (2006).

${ }^{9}$ W. Guan, R. Fan, and M. A. Reed, Nat. Commun. 2, 506 (2011).
${ }^{10}$ M. Ali, P. Ramirez, S. Mafe, R. Neumann, and W. Ensinger, ACS Nano 3, 603 (2009).

${ }^{11}$ W. Guo, L. Cao, J. Xia, F.-Q. Nie, W. Ma, J. Xue, Y. Song, D. Zhu, Y. Wang, and L. Jiang, Adv. Funct. Mater. 20, 1339 (2010).

${ }^{12}$ P. Ramirez, M. Ali, W. Ensinger, and S. Mafe, Appl. Phys. Lett. 101, 133108 (2012).

${ }^{13}$ E. C. Yusko, R. An, and M. Mayer, ACS Nano 4, 477 (2010).

${ }^{14}$ S. Lee, Y. Zhang, H. S. White, C. C. Harrell, and C. R. Martin, Anal. Chem. 76, 6108 (2004).

${ }^{15}$ R. J. White and H. S. White, Anal. Chem. 79, 6334 (2007).

${ }^{16}$ E. M. Nestorovich, C. Danelon, M. Winterhalter, and S. M. Bezrukov, Proc. Natl. Acad. Sci. U.S.A. 99, 9789 (2002).

${ }^{17}$ S. Mafe, P. Ramírez, and A. Alcaraz, J. Chem. Phys. 119, 8097 (2003).

${ }^{18}$ V. A. Karginov, E. M. Nestorovich, M. Moayeri, S. H. Leppla, and S. M. Bezrukov, Proc. Natl. Acad. Sci. U.S.A. 102, 15075 (2005).

${ }^{19}$ M. Aguilella-Arzo, J. Cervera, P. Ramirez, and S. Mafe, Phys. Rev. E 73, 041914 (2006).

${ }^{20}$ C. Verdiá-Báguena, M. Queralt-Martín, V. M. Aguilella, and A. Alcaraz, J. Phys. Chem. C 116, 6537 (2012).

${ }^{21}$ R. D. Astumian, J. C. Weaver, and R. K. Adair, Proc. Natl. Acad. Sci. U.S.A. 92, 3740 (1995).

${ }^{22}$ J. A. Manzanares, J. Cervera, and S. Mafe, Appl. Phys. Lett. 99, 153703 (2011).

${ }^{23}$ D. J. Blackiston, K. A. McLaughlin, and M. Levin, Cell Cycle 8, 3527 (2009).

${ }^{24}$ M. Levin and C. G. Stevenson, Annu. Rev. Biomed. Eng. 14, 295 (2012).

${ }^{25}$ M. Davenport, K. Healy, M. Pevarnik, N. Teslich, S. Cabrini, A. P. Morrison, Z. S. Siwy, and S. E. Létant, ACS Nano 6, 8366 (2012).

${ }^{26}$ P. Y. Apel, I. V. Blonskaya, O. L. Orelovitch, B. A. Sartowska, and R. Spohr, Nanotechnology 23, 225503 (2012).

${ }^{27}$ M. Wanunu, W. Morrison, Y. Rabin, A. Y. Grosberg, and A. Meller, Nat. Nanotechnol. 5, 160 (2010).

${ }^{28}$ M. X. Macrae, S. Blake, M. Mayer, and J. Yang, J. Am. Chem. Soc. 132, 1766 (2010).

${ }^{29}$ M. Tagliazucchi, Y. Rabin, and I. Szleifer, ACS Nano 7, 9085 (2013).

${ }^{30}$ M. Tsutsui, Y. Maeda, Y. He, S. Hongo, S. Ryuzaki, S. Kawano, T. Kawai, and M. Taniguchi, Appl. Phys. Lett. 103, 013108 (2013).

${ }^{31}$ Z. Siwy and A. Fuliński, Am. J. Phys. 72, 567 (2004).

${ }^{32}$ E. Kalman, K. Healy, and Z. S. Siwy, Europhys. Lett. 78, 28002 (2007).

${ }^{33}$ M. Ali, S. Nasir, P. Ramirez, J. Cervera, S. Mafe, and W. Ensinger, J. Phys. Chem. C 117, 18234 (2013). 